\title{
Dural Venous Sinus Stenosis: Why Distinguishing Intrinsic- versus-Extrinsic Stenosis Matters
}

\author{
(D) S.H. Sundararajan, (D) A.D. Ramos, (D) V. Kishore, (D) M. Michael, (D) R. Doustaly, (D)F. DeRusso, and (D) A. Patsalides
}

\begin{abstract}
BACKGROUND AND PURPOSE: Dural venous sinus stenosis has been associated with idiopathic intracranial hypertension and isolated venous pulsatile tinnitus. However, the utility of characterizing stenosis as intrinsic or extrinsic remains indeterminate. The aim of this retrospective study was to review preprocedural imaging of patients with symptomatic idiopathic intracranial hypertension and pulsatile tinnitus, classify the stenosis, and assess a trend between stenosis type and clinical presentation while reviewing the frequencies of other frequently seen imaging findings in these conditions.
\end{abstract}

MATERIALS AND METHODS: MRVs of 115 patients with idiopathic intracranial hypertension and 43 patients with pulsatile tinnitus before venous sinus stent placement were reviewed. Parameters recorded included the following: intrinsic or extrinsic stenosis, prominent emissary veins, optic nerve tortuosity, cephalocele, sella appearance, poststenotic fusiform enlargement versus saccular venous aneurysm, and internal jugular bulb diverticula. $\chi^{2}$ cross-tabulation statistics were calculated and recorded for all data.

RESULTS: Most patients with idiopathic intracranial hypertension (75 of 115 sinuses, 65\%) had extrinsic stenosis, and most patients with pulsatile tinnitus (37 of 45 sinuses, $82 \%$ ) had intrinsic stenosis. Marked optic nerve tortuosity was more common in idiopathic intracranial hypertension. Cephaloceles were rare in both cohorts, with an increased trend toward the presence in idiopathic intracranial hypertension. Empty sellas were more common in idiopathic intracranial hypertension. Cerebellar tonsils were similarly located at the foramen magnum level in both cohorts. Saccular venous aneurysms were more common in pulsatile tinnitus. Internal jugular bulb diverticula were similarly common in both cohorts.

CONCLUSIONS: In this cohort, most patients with idiopathic intracranial hypertension had extrinsic stenosis, and most patients with pulsatile tinnitus had intrinsic stenosis. Awareness and reporting of these subtypes may reduce the underrecognition of potential contributory stenoses in a given patient's idiopathic intracranial hypertension or pulsatile tinnitus.

diopathic intracranial hypertension has undergone several name changes since the 1890 s, previously referred to pseudotumor cerebri, followed by benign intracranial hypertension, and, most recently, idiopathic intracranial hypertension. While the term "idiopathic" is used to describe this pathology, there has been continued emergence of research pointing to idiopathic

Received June 8, 2020; accepted after revision September 4.

From the Department of Neurosurgery (S.H.S., A.D.R., M.M., F.D.), Division of Interventional NeuroRadiology, NY Presbyterian Hospital Weill Cornell Medicine, New York, New York; GE Healthcare (V.K., R.D.), Buc, France; and Department of Neuro-Interventional Surgery (A.P.), North Shore University Hospital, Northwell Health, Manhasset, New York.

Data from this article were first presented as an ePoster and recorded lecture at: Annual Meeting of the American Society of Neuroradiology, May 30 to June 4, 2020; Virtual.

Please address correspondence to Athos Patsalides, MD, MPH, NeuroInterventional Surgery, North Shore University Hospital, Northwell Health, 300 Community Dr, Manhasset, NY 11030; e-mail: apatsalides@northwell.edu; @radiolosri

- Indicates open access to non-subscribers at www.ajnr.org http://dx.doi.org/10.3174/ajnr.A6890 intracranial hypertension stemming from a neurovascular etiology. The redistribution pathway of CSF involves absorption from brain and spinal subarachnoid spaces into the systemic circulation via arachnoid granulations located within the walls of the dural venous sinuses. Thus, blockage or severe stenoses of the dural venous sinuses responsible for draining intracranial venous circulation has the potential to limit CSF re-absorption, therefore leading to gradual intracranial CSF buildup with subsequent elevations of intracranial pressure and resultant symptomology as reported in the literature. ${ }^{1-4}$

Although pulsatile tinnitus has been associated with idiopathic intracranial hypertension, its unique manifestation in either the setting of idiopathic intracranial hypertension or in isolation remains a clinical enigma. This vascular somatosound has been reported in the setting of venous sinus stenosis, lateral sinus wall dehiscence with associated venous sinus aneurysms, and prominent collateral circulation via mastoid, condylar, or occipital emissary veins. ${ }^{5,6}$

Dural venous sinus stenosis can be characterized as either intrinsic or extrinsic stenosis. Intrinsic stenosis refers to internal 


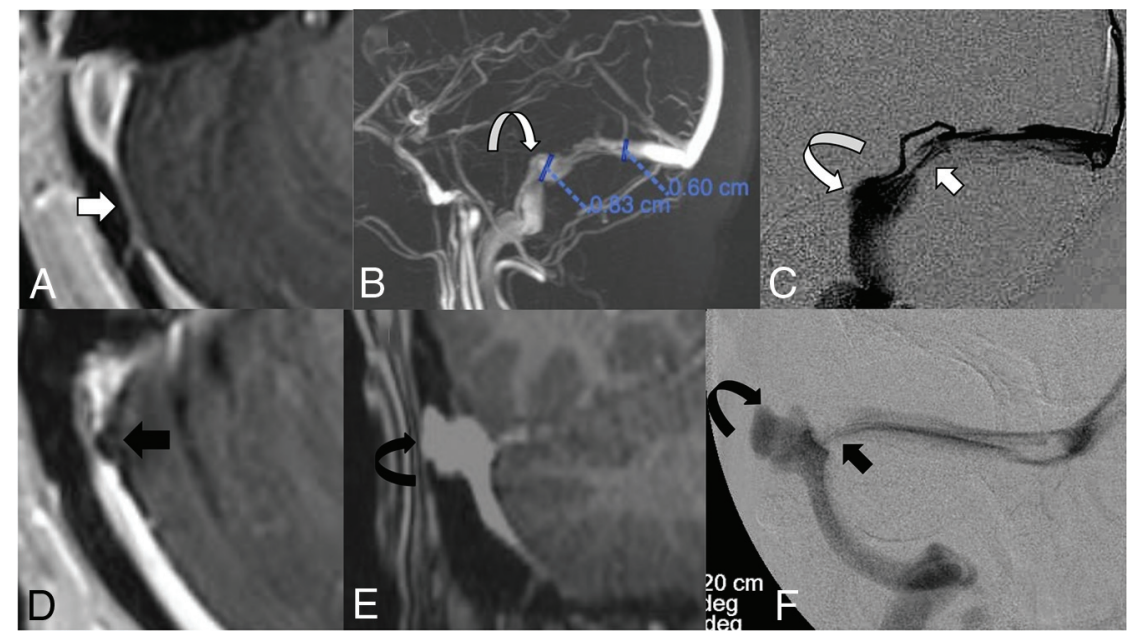

FIG 1. A, Axial postcontrast MRV demonstrating extrinsic stenosis from the overlying cerebellum (short white arrow). B, Contrast-enhanced 3D-MRV image shows poststenotic sigmoid sinus enlargement (curved white arrow). C, Accompanying lateral venography confirms stenosis (white arrow) and sinus enlargement (curved white arrow) seen on the corresponding MRV. D, Separate axial postcontrast MRV shows intrinsic stenosis from arachnoid granulations (black arrow). E, A coronal postcontrast MRV sequence shows lateral sinus dehiscence with a venous aneurysm (curved black arrow). F, Accompanying frontal venography confirms stenosis (short black arrow) and a saccular aneurysm (curved black arrow) seen on the corresponding MRV.

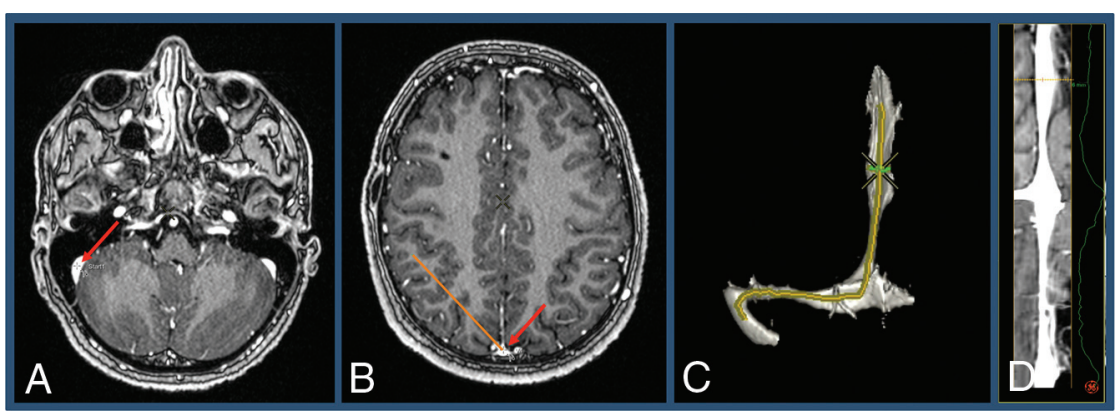

FIG 2. A, 2-Click automatic vessel analysis start point selection in the sigmoid sinus (short arrow). B, 2-Click automatic vessel analysis end point selection (short arrow) in the superior sagittal sinus (long arrow). C, 3D volume-rendered vessel segmentation. D, Lumen view shows the straightened vessel segmentation.

filling defects such as prominent arachnoid granulations that narrow the sinus. Extrinsic stenosis involves external compression of the sinus by adjacent brain parenchyma. ${ }^{7,8}$ Despite the overlap of venous sinus stenosis in both idiopathic intracranial hypertension and isolated pulsatile tinnitus of venous etiology, the type of stenosis is seldom distinguished in clinical practice despite a pre-existing classification schema in the literature. The purpose of this study was to review preprocedural imaging of treated patients with symptomatic idiopathic intracranial hypertension and pulsatile tinnitus in our institution and classify the stenosis to assess the presence or absence of a trend between stenosis type and clinical presentation.

\section{MATERIALS AND METHODS}

Following institutional review board (Weill Cornell Medicine) approval, a retrospective review of patients treated with venous sinus stent placement from January 2012 to January 2020 was performed. Two certified neuroradiologists reviewed, in consensus, MRV head imaging performed within 3 months of a planned intervention and conventional venography performed at the time of the subsequent intervention. All institutional MRV examinations were performed on 3T units (Magnetom Skyra; Siemens; and Signa Architect; GE Healthcare) with coronal 2D-TOF, sagittal 3D phase contrast, and 3D thin-section T1 pre- and postcontrast sequences (echo-spoiled gradient echo or MPRAGE). Contrast-enhanced sequences were acquired following weight-based intravenous administration of gadobutrol (Gadavist; Bayer Schering Pharma). Classification schemas were implemented for all recorded imaging findings to allow the objective recording of parameters and subsequent data evaluation. $\chi^{2}$ cross-tabulation statistics were calculated and recorded for all data, with an $\alpha$ of .05 used for all computations.

Patients were categorized into the idiopathic intracranial hypertension and isolated venous pulsatile tinnitus cohorts before planned interventions. Eligibility for idiopathic intracranial hypertension cohort categorization required a diagnosis of idiopathic intracranial hypertension as per pre-established criteria, including a lumbar puncture opening pressure of $\geq 25 \mathrm{~cm}$ $\mathrm{H}_{2} \mathrm{O}$ in the left lateral decubitus position and preprocedural clinical evaluation for papilledema by an ophthalmologist. ${ }^{3}$

Categorization into the pulsatile tinnitus cohort consisted of several strict criteria. Patients with clinical findings consistent with pulsatile tinnitus from a venous origin and imaging findings of lateral venous sinus stenosis of at least $50 \%$ either ipsilateral to the pulsatile tinnitus or within the dominant sinus were included in the pulsatile tinnitus cohort. Patients with causes of pulsatile tinnitus other than lateral sinus stenosis were excluded. An otorhinolaryngologist evaluated all patients within 6 months of planned interventions. Patients with otologic disorders, nonpulsatile tinnitus, or spontaneous CSF leak were excluded. Similarly, an ophthalmologist evaluated all patients within 6 months of planned interventions. The presence of papilledema of any degree was considered an exclusion criterion. Patients with venous pulsatile tinnitus symptomology yet with opening pressure of $25 \mathrm{~cm} \mathrm{H}_{2} \mathrm{O}$ or higher were excluded from the pulsatile tinnitus cohort and instead were included in the idiopathic intracranial hypertension cohort. Patients with opening pressure between 20 and $24 \mathrm{~cm} \mathrm{H}_{2} 0$ were 


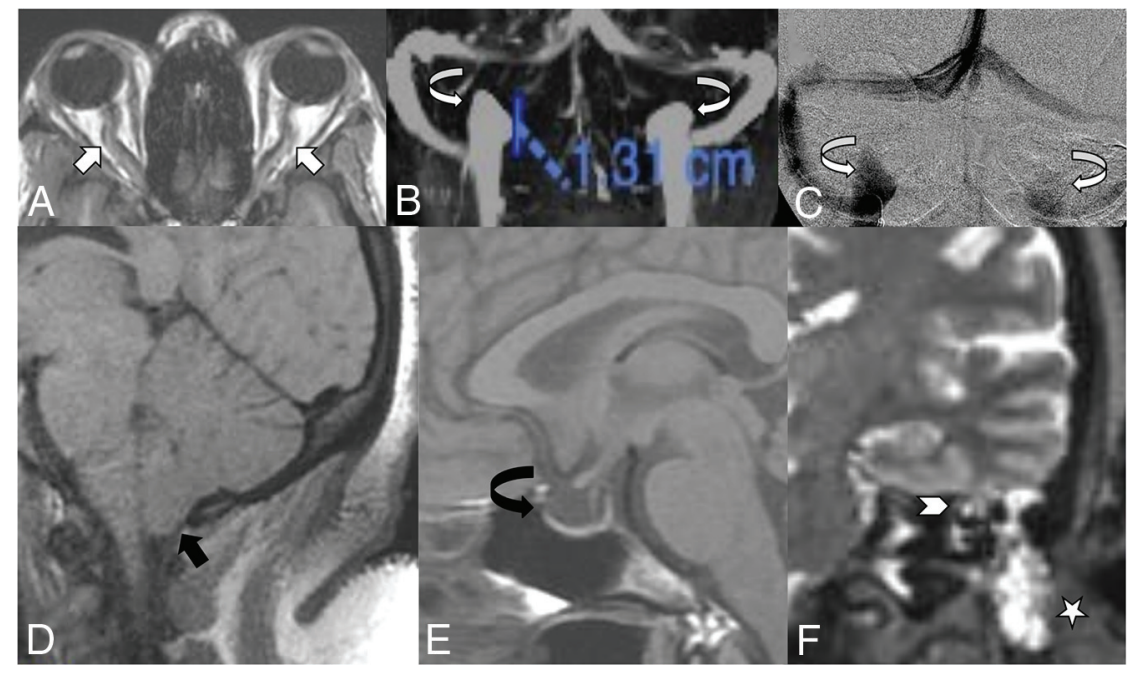

FIG 3. Objective parameters implemented in recording corollary findings of both idiopathic intracranial hypertension and pulsatile tinnitus cohorts. A, Marked optic nerve tortuosity, with $>50 \%$ of optic sheath width deviation noted relative to its expected straight path along the optic canal (white arrows). Bilateral $\geq 5$-mm internal jugular bulb diverticula, as seen on MRV (B) and catheter venography $(C)$ images (curved white arrows). D, Cerebellar tonsil projecting 1$3 \mathrm{~mm}$ below the foramen magnum, referred to as ectopia (black arrow). E, Empty sella recorded if there is $>75 \%$ loss of pituitary height (curved black arrow). F, Coronal T2 MR imaging demonstrates a left temporal lobe cephalocele through the tegmen tympani (arrowhead) and CSF in mastoid air cells (star).

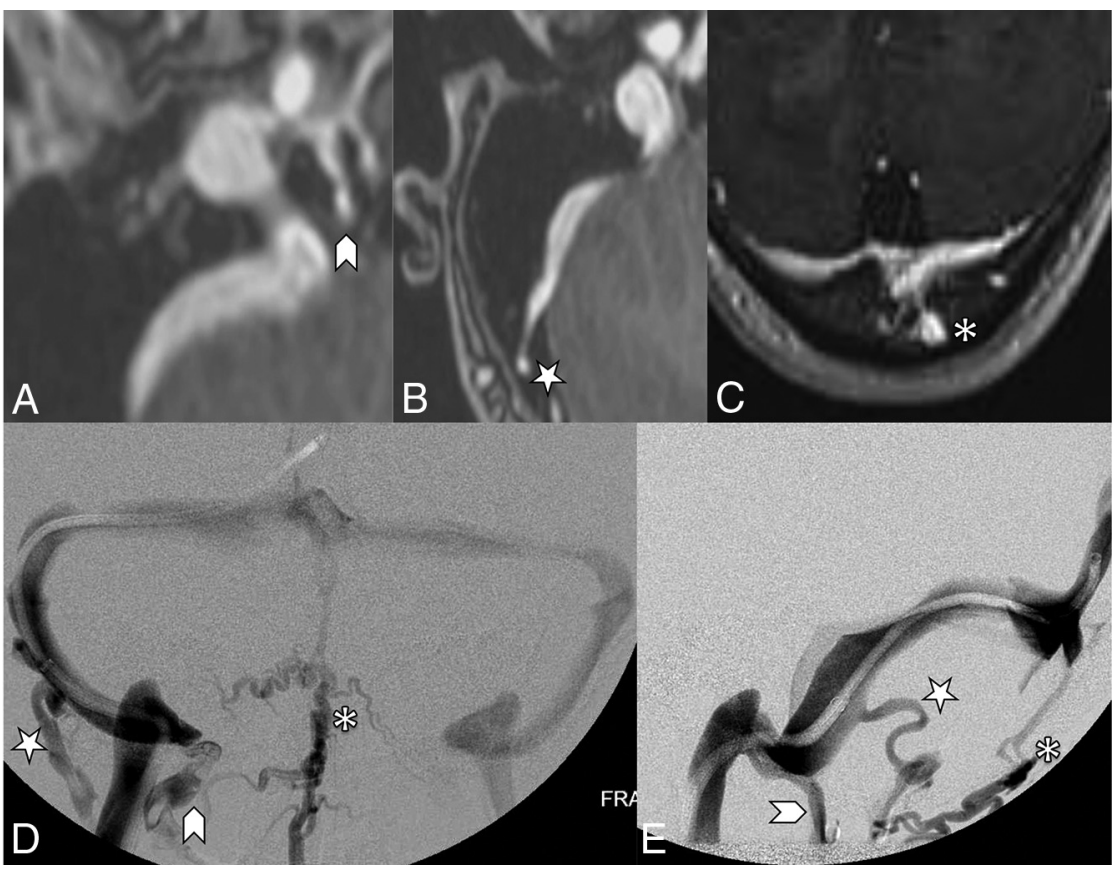

FIG 4. Contrast-enhanced MRV images $(A, B$, and $C)$ highlighting features of the emissary veins categorized in this study. $A, A$ condylar vein is seen arising from the internal jugular vein bulb extending through the condylar canal (arrowhead). B, A mastoid emissary vein is seen arising from the sigmoid sinus traversing the mastoid foramen (star). $C$, An occipital emissary vein is seen arising from the torcula extending through the calvaria (asterisk). Conventional venography frontal $(D)$ and lateral $(E)$ images showcase the 3 emissary vein types categorized in this study. The condylar vein (arrowhead) extends inferiorly toward the vertebral plexus. The mastoid emissary vein (star) extends posteriorly and inferiorly to join the suboccipital plexus and external jugular vein. The occipital emissary vein (asterisk) drains inferiorly into the suboccipital plexus. initially medically managed with acetazolamide for 4 weeks with subsequent inclusion in the pulsatile tinnitus cohort if there was no major improvement of pulsatile tinnitus. The severity of pulsatile tinnitus was assessed with a standardized questionnaire, the Tinnitus Handicap Inventory. ${ }^{9}$ A minimum score of 38 , consistent with moderate severity, was required for inclusion in the pulsatile tinnitus cohort.

All venous sinus stenoses included were considered severe at the time of initial and retrospective study review. The location and type of stenosis were recorded on the basis of findings made from MRV examinations. However, because all cohort patients underwent venous sinus stent placement, conventional venography images were available for review and useful in corroborating the stenosis subtype characterized on MRV. Stenosis lengths were not recorded. Stenosis caused by intraluminal processes such as prominent arachnoid granulations were classified as intrinsic stenosis. Stenosis caused by compression of the venous sinus from brain parenchyma against the adjacent calvaria was classified as extrinsic stenosis. In the event of intrinsic stenoses preceding segments of extrinsic stenosis, the overall stenosis was still categorized as extrinsic stenosis. The presence of poststenotic dilation of the lateral sinus was classified as "fusiform" if it had the appearance of a shallow, wide-neck aneurysm with a $25 \%-50 \%$ increase in size relative to its expected diameter. The term "saccular" was used if it had the appearance of a discrete dome with the depth larger than the neck dimensions. Examples of these findings are shown in Fig 1.

Straight-vessel views and 3D reconstructions of both TOF and contrastenhanced images were created and reviewed to better characterize venous sinus anatomy and stenosis, as shown in Fig 2. The straightened vessel view was achieved using 2-click automatic vessel analysis (Vessel ASSIST; GE Healthcare). After the user selected the start and end points in the sigmoid sinus and superior sagittal sinus, the software automatically tracked the vessel and computed its centerline. The resulting vessel segmentation could be displayed 
Table 1: Idiopathic intracranial hypertension and pulsatile tinnitus cohort demographics

\begin{tabular}{lcc}
\hline Demographic & $\begin{array}{c}\text { Idiopathic Intracranial } \\
\text { Hypertension }\end{array}$ & $\begin{array}{c}\text { Pulsatile } \\
\text { Tinnitus }\end{array}$ \\
\hline No. of patients & 115 & 43 \\
Age (yr) & Mean $=32.8$ & Mean $=38.1$ \\
& Min $=4$ & Min $=20$ \\
Max $=66$ & Max $=67$ \\
No. of females & $107(90 \%)$ & $41(95 \%)$ \\
No. of males & $8(10 \%)$ & $2(5 \%)$ \\
No. of sinuses: & 115 & 45 sinuses $^{\mathrm{a}}$ \\
Right & 90 & 29 \\
Left & 25 & 16 \\
\hline
\end{tabular}

Note:-Min indicates minimum; Max, maximum.

${ }^{\mathrm{a}}$ Two patients were stented bilaterally.

Table 2: Breakdown of intrinsic and extrinsic stenosis relative to the total number of sinuses and distribution of additional corollary findings noted in the idiopathic intracranial hypertension and pulsatile tinnitus cohorts ${ }^{a}$

\begin{tabular}{lrr}
\hline \multicolumn{1}{c}{ Imaging Finding } & IIH & PT \\
\hline Stenosis type & & \\
Extrinsic stenosis & 58 & 8 \\
Mixed extrinsic and intrinsic & 17 & 0 \\
Intrinsic stenosis & 40 & 37 \\
Total & 115 & 45 \\
$\chi^{2} P$ value $\leq .001^{b}$ & & \\
Saccular venous aneurysm & & \\
Yes & 4 & 11 \\
No & 111 & 34 \\
Total & 115 & 45 \\
$\chi^{2} P$ value $\leq .001$ & & \\
Sella appearance & & 18 \\
Partially empty & 49 & 11 \\
Empty & 61 & 1 \\
Normal & 5 & \\
NA (adenoma) & 0 & \\
Total & 115 & 31 \\
$\chi^{2} P$ value $\leq .001$ & & 14 \\
Optic nerve tortuosity & & \\
Minimal & 46 \\
Marked & 69 & \\
Total & \\
$\chi^{2} P$ value $=.001$ & 115 & \\
\hline
\end{tabular}

Note:-NA indicates not applicable; IIH, idiopathic intracranial hypertension; PT, pulsatile tinnitus.

${ }^{a} \chi^{2}$ cross-tabulation $P$ values were calculated for each variable. Eighty-two percent of pulsatile tinnitus sinuses had intrinsic stenosis, and $65 \%$ of idiopathic intracranial hypertension sinuses had extrinsic stenosis $(P$ value $<.001)$. Statistically significant differences were noted when comparing the distribution of optic nerve tortuosity, sella appearance, and saccular aneurysm presence between the 2 groups ( $P$ values $\leq$.001).

${ }^{\mathrm{b}}$ Extrinsic and mixed subgroups summed for $\chi^{2}$ calculation.

as a $3 \mathrm{D}$ volume-rendered model or a lumen view (D). The lumen view is obtained by transforming the $3 \mathrm{D}$ vessel centerline into a straight line and displaying the corresponding perpendicular plane for each point on the line. For each position, minimum, mean, and maximum diameters were automatically computed.

The other recorded imaging findings included the presence or absence of prominent emissary veins, optic nerve tortuosity, cephalocele, sella appearance, sinus enlargement versus aneurysm, and internal jugular bulb diverticula as shown in Fig 3. The presence of prominent emissary veins was initially detected on MRV and corroborated on subsequent conventional venography.
Table 3: Continued breakdown of intrinsic and extrinsic stenosis relative to the total number of sinuses and distribution of additional corollary findings noted in the idiopathic intracranial hypertensin and pulsatile tinnitus cohorts ${ }^{a}$

\begin{tabular}{lrr}
\hline \multicolumn{1}{c}{ Imaging Finding } & IIH & PT \\
\hline Internal jugular bulb diverticulum size & & \\
$<5 \mathrm{~mm}$ & 47 & 26 \\
$\geq 5 \mathrm{~mm}$ & 68 & 19 \\
Total & 115 & 45 \\
$\chi^{2} P$ value $=.053$ & & \\
Cerebellar tonsil location & & \\
Above & 22 & 11 \\
At & 54 & 23 \\
Ectopia & 35 & 11 \\
Above (prior Chiari I surgery) & 4 & 0 \\
Total & 115 & 45 \\
$\chi^{2} P$ value $=.522$ & & \\
Cephalocele presence & & \\
No & 110 & 44 \\
Yes & 5 & 1 \\
Total & 115 & 45 \\
$\chi^{2}$ P value = .525 & & \\
Emissary vein prominence & & \\
Condylar & 52 & 19 \\
Occipital and condylar & \\
Mastoid & 28 & 11 \\
Mastoid and condylar & 6 & 1 \\
Occipital, condylar, and mastoid & 5 & 3 \\
None & 1 & 2 \\
Total & 23 & 9 \\
$\chi^{2} P$ value = 1 & 115 & 45 \\
\hline
\end{tabular}

Note:- IIH indicates idiopathic intracranial hypertension; PT, pulsatile tinnitus. ${ }^{a}$ No significant differences were noted when comparing the distribution of internal jugular bulb diverticulum size, cerebellar tonsil location, cephalocele pres ence, or emissary vein prominence ( $P$ values $\geq .053)$.

The 3 subtypes classified were condylar arising from the internal jugular bulb, mastoid arising from the sigmoid sinus, and occipital arising from the torcula as shown in Fig 4.

Optic nerve tortuosity was evaluated on axial thin-section T1and $\mathrm{T} 2$-weighted images when available. Tortuosity was classified as marked if the optic sheath width deviated $>50 \%$ of its diameter relative to its expected straight path from the optic canal and minimal if $<50 \%$ of its diameter. Cephaloceles were characterized as being either present or absent.

The degree of mass effect on the sella was graded in reference to the noncompressed posterior pituitary gland relative to the expected pituitary gland height in each case. The sella was characterized as empty if $>75 \%$ of pituitary parenchymal height was lost on sagittal plane imaging and partially empty if anywhere between $25 \%$ and $75 \%$ loss of pituitary height was identified. The cerebellar tonsils were graded according to location on sagittal plane imaging. 'Above' was used when both cerebellar tonsils were above the foramen magnum, noting 4 separate patients with prior Chari 1 surgical intervention were separately categorized. 'At' was used when one or both cerebellar tonsils were at the foramen magnum. 'Ectopia' was used when one or both cerebellar tonsils were projecting below the foramen magnum by $1-3 \mathrm{~mm}$. Internal jugular bulb diverticula were evaluated on coronal plane MRV imaging, with sizes graded according to height projecting above a line drawn perpendicular to the distal sigmoid sinus insertion into the internal jugular vein. Diverticula were 


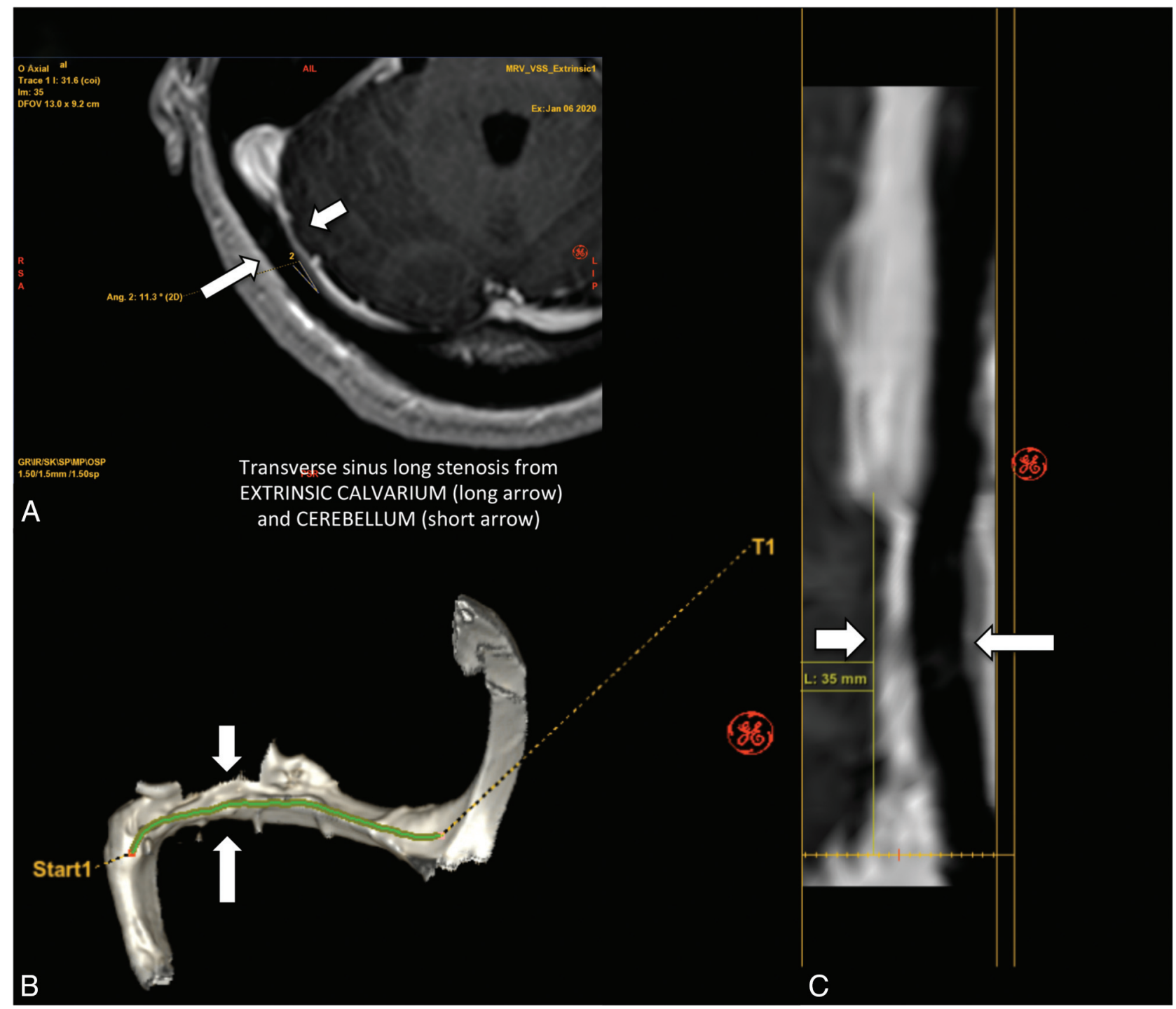

FIG 5. MR images showing extrinsic stenosis of the right transverse sinus. The short arrows point to right cerebellar parenchyma location, and the long arrows points to the occipital calvaria location. A, An axial contrast-enhanced MRV image. B, A 3D reconstruction image. C, A straightvessel reformat of the right transverse-to-proximal sigmoid sinus from source contrast-enhanced axial images.

categorized as either being small $(<5 \mathrm{~mm}$ in height) or prominent ( $\geq 5 \mathrm{~mm}$ in height).

\section{RESULTS}

The imaging of 115 patients with idiopathic intracranial hypertension (107 women; mean, 32.8 years age of age) and 43 with pulsatile tinnitus (41 women; mean, 38.1 years of age) who underwent subsequent endovascular intervention was reviewed. Forty-five sinuses were counted in the pulsatile tinnitus cohort because 2 patients were bilaterally stented (Tables 1-3). Regardless of the cohort, all patients with intrinsic stenosis had either a single arachnoid granulation or a cluster of prominent arachnoid granulations located at the transverse-sigmoid sinus junction. Similarly, all patients with extrinsic stenosis in both cohorts were from overlying brain parenchyma extending from the transverse sinus to the sigmoid sinus.

In both idiopathic intracranial hypertension and pulsatile tinnitus cohorts, most patients similarly had prominence of the emissary veins $(P=1)$. The 2 most commonly seen were condylar and occipital subtypes. Marked optic nerve tortuosity was more common in the idiopathic intracranial hypertension cohort compared with the pulsatile tinnitus cohort $(P=.001)$. Cephaloceles were infrequently present in both cohorts, with an increased trend toward their presence in the idiopathic intracranial hypertension cohort relative to the pulsatile tinnitus cohort $(P=.525)$. Empty sellas were more common in the idiopathic intracranial hypertension cohort compared with the pulsatile tinnitus cohort $(P<.001)$. Cerebellar tonsils were frequently located at the foramen magnum level in both cohorts $(P=.522)$. Saccular aneurysms were more common in the pulsatile tinnitus cohort relative to the idiopathic intracranial hypertension cohort $(P<.001)$. Internal jugular bulb diverticula were similarly common in distribution across both cohorts, regardless of $<5$ or $\geq 5 \mathrm{~mm}$ size $(P=.053)$. Specifics regarding these findings are noted in Fig 4.

Seventy-five of 115 (65\%) sinuses in the idiopathic intracranial hypertension cohort had extrinsic stenosis related to 


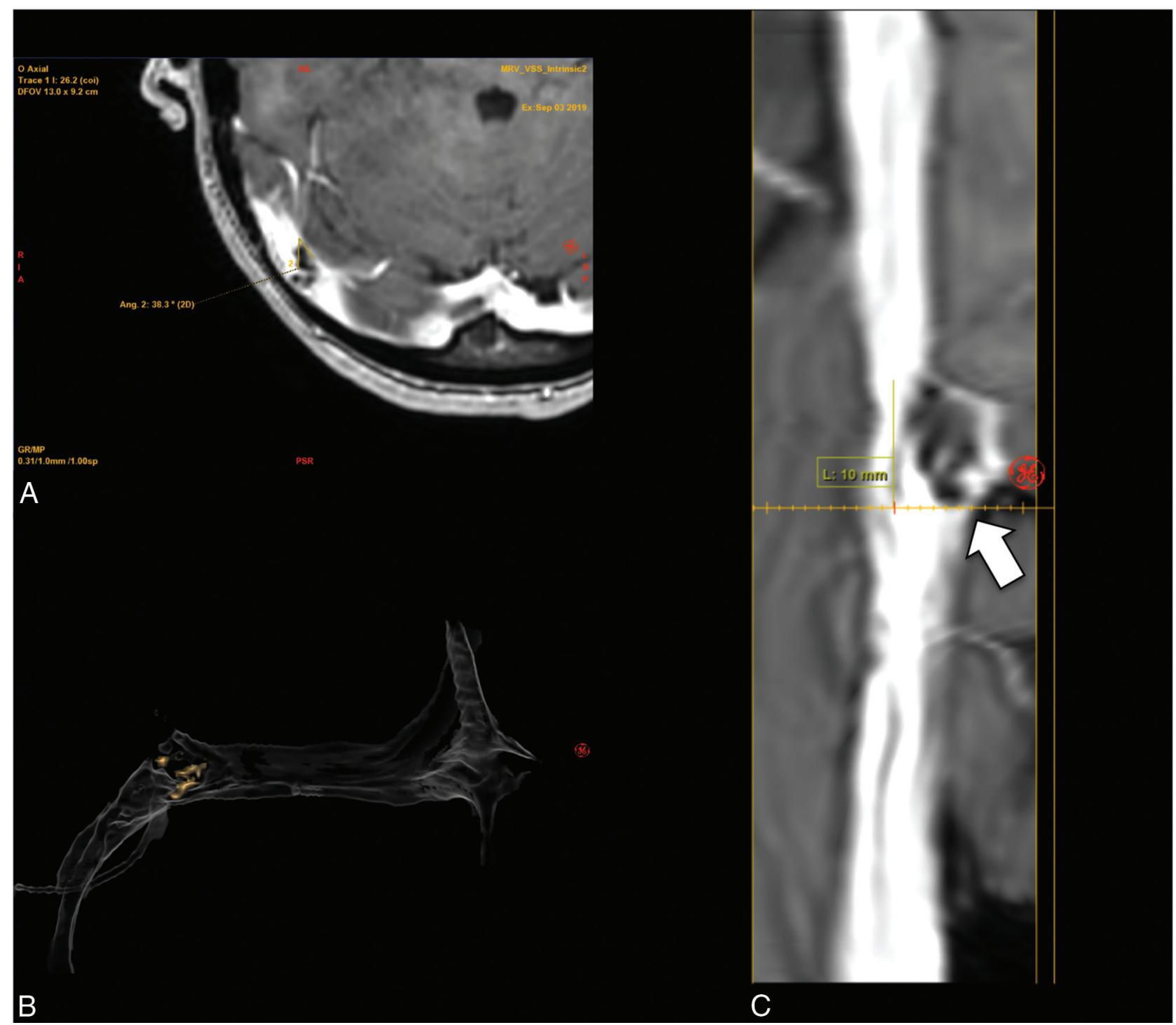

FIG 6. MR images showing intrinsic stenosis of the right transverse sinus. The short arrow points to a prominent arachnoid granulation situated inside the sinus. A, An axial contrast-enhanced MRV image, $B, A$ S reconstruction image. $C, A$ straight-vessel reformat of the right transverseto-proximal sigmoid sinus from source contrast-enhanced axial images, noting orange shading of the arachnoid granulation and transparency of the remaining dural venous sinus.

narrowing from adjacent parenchyma. Seventeen of these sinuses were in patients with dominant extrinsic stenosis and superimposed prominent arachnoid granulations flanking either end of the extrinsic stenosis. The remaining 58 sinuses had extrinsic stenosis alone. Forty patients in the idiopathic intracranial hypertension cohort (35\%) had intrinsic stenosis related to arachnoid granulations.

Thirty-seven of $45(82 \%)$ sinuses in the pulsatile tinnitus cohort had intrinsic stenosis related to arachnoid granulations. In the remaining 8 patients in the pulsatile tinnitus cohort, all 8 (18\%) had extrinsic stenosis related to adjacent brain parenchyma. As noted above, 11 of 45 (24\%) had saccular venous aneurysms of the transverse/sigmoid junction. The remaining 34 of 45 (76\%) had poststenotic fusiform sinus enlargement.

The proportion of patients with idiopathic intracranial hypertension presenting with extrinsic stenosis relative to those with pulsatile tinnitus presenting with intrinsic stenosis was a statistically significant difference $(P$ value $<.001)$. These results are summarized in Tables 2 and 3.

\section{DISCUSSION}

In this cohort, review of neuroimaging demonstrated intrinsic and extrinsic stenosis presence in the transverse sinus, transversesigmoid sinus junction, and proximal sigmoid sinus in patients with both idiopathic intracranial hypertension and isolated pulsatile tinnitus clinical symptomology. Extrinsic and intrinsic stenoses alike were best detected on thin-section contrast-enhanced axial MRV images, reconstructed straight-vessel views of the affected dural venous sinus, and 3D reconstructions of either TOF or contrast-enhanced MRV imaging (Figs 5 and 6). Within the idiopathic intracranial hypertension cohort, $65 \%$ of the sinuses had extrinsic stenosis about the margins of the transverse and sigmoid sinuses. This finding is in contrast to the pulsatile tinnitus cohort in which $82 \%$ of the sinuses had intrinsic stenosis related to arachnoid granulations, noting that the presence or absence of superimposed webbing may contribute to stenosis as well. The imaging features of webbing or intraluminal synechia are ill-defined on cross-sectional imaging modalities, noting that 


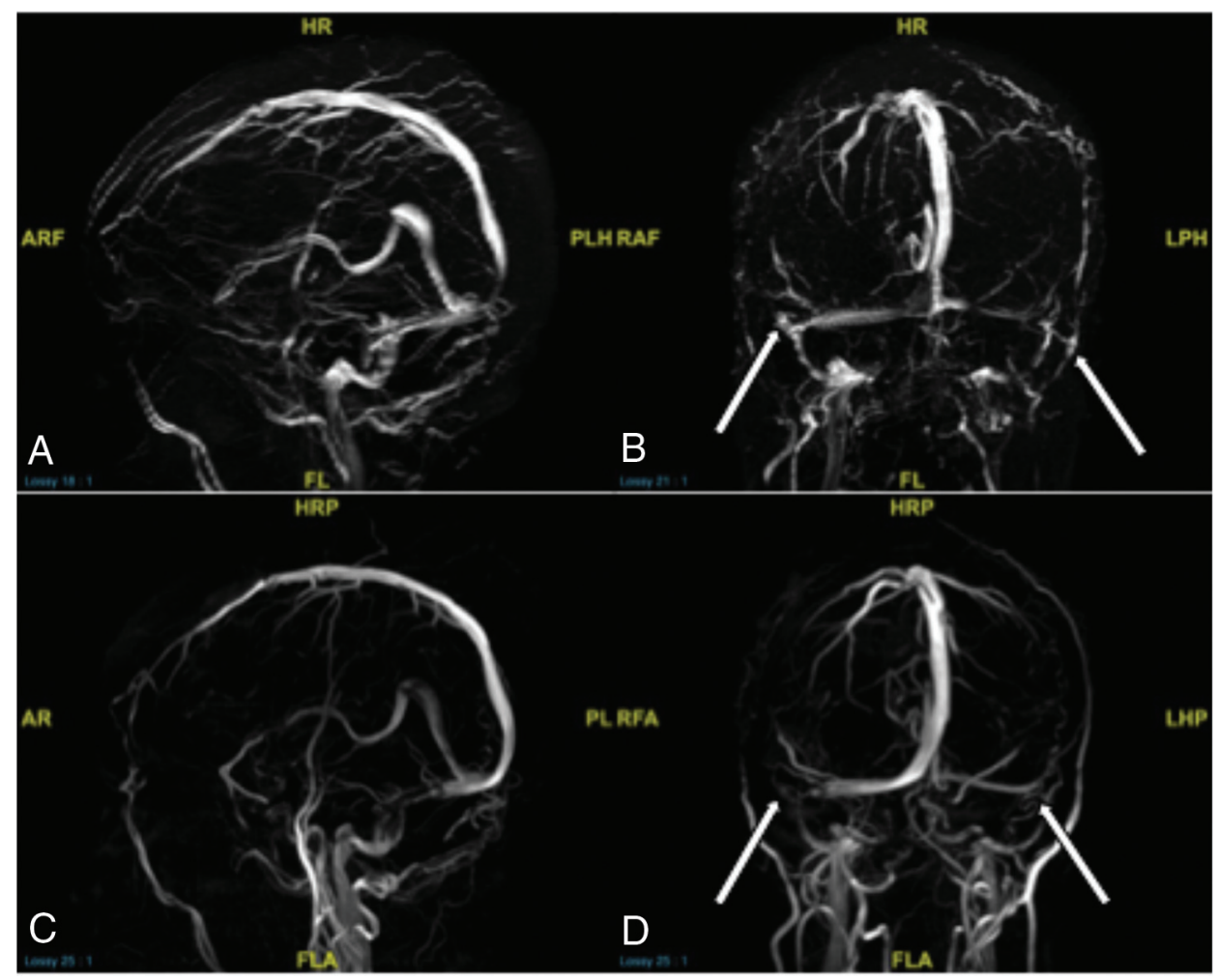

FIG 7. Comparing TOF sagittal and coronal 3D reconstructions $(A$ and $B)$ and contrast-enhanced sagittal and coronal 3D reconstructions ( $C$ and $D)$ from MRV in a patient with idiopathic intracranial hypertension. Note how the TOF images show the patient's physiologic venous drainage due to properties of TOF imaging, showing only blood draining back to the patient's heart (veins). Contrast-enhanced imaging, though crisper, shows arteries and veins in the same image. $B$ and $D$, Arrows point to severe extrinsic stenoses in the bilateral transverse sinus-sigmoid sinus junctions, a common location for idiopathic intracranial hypertension stenosis. Such short-segment severe stenoses appear to simulate the abrupt narrowing commonly seen in short-segment intrinsic stenoses on these 3D reconstructions. However, review of source imaging would demonstrate brain parenchymal narrowing rather than primary arachnoid granulations producing the stenoses. HRP indicates head right posterior; PLH, posterior left head; RAF, right anterior foot; RA, right anterior; FL, foot left; PL, posterior left; RFA, right foot anterior; LHP, left head posterior; LPH, left posterior head; ARF, anterior right foot; AR, anterior right; FLA, foot left anterior; AF, anterior foot.

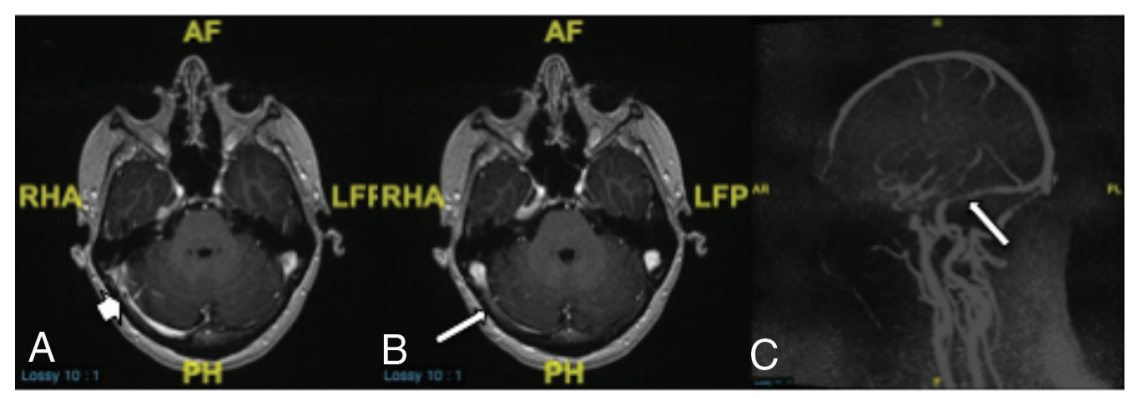

FIG 8. MR images demonstrating focal intrinsic stenosis just proximal to dominant extrinsic stenosis in this patient with idiopathic intracranial hypertension. The short arrow in $A$ demonstrates focal arachnoid granulation. The long arrows in $B$ and $C$ demonstrate a primary extrinsic stenosis pattern. Despite the mixed presence of intrinsic and extrinsic stenoses, this was categorized as primary extrinsic stenosis. RHA indicates right head anterior; $\mathrm{PH}$, posterior head; LFP, left foot posterior; $\mathrm{AF}$, anterior foot; $\mathrm{AR}$, anterior right; $\mathrm{PL}$, posterior left; $\mathrm{H}$, head; $\mathrm{F}$, foot.

their presence can be detected with the use of intravascular ultrasound. ${ }^{10}$ However, in our experience, knowledge of the presence or absence of webbing with an otherwise MR imaging-evident stenosis produced by arachnoid granulation or brain parenchyma was not beneficial in procedural planning. Thus, the use of intravascular ultrasound during venous sinus procedures is no longer routinely performed at our institution.

MRV without contrast involves the use of TOF techniques that image the flow of blood in a specific direction, therefore allowing its specificity in looking at the venous system rather than the arteries. TOF allows a physiologic approximation of the dural venous sinus caliber in relation to venous flow through the sinus that can better approximate the true degree of stenosis in a given segment. By administering contrast, one can better characterize a stenosis as the sinus lining and can inspect luminal content with a high special resolution compared with flow-related techniques alone. Contrastenhanced images also provide the true sinus caliber relative to surrounding calvarial demarcations, an important distinction compared with flow when measuring for hardware sizes, depending on the dural venous sinus segment to be stented. Volume-rendered and 3D reconstructed images from source TOF and contrastenhanced images were of equal importance in demonstrating a global understanding of the type and location of stenosis (Fig 7). ${ }^{10,11}$

Confounders in the dichotomous characterization of intrinsic-versus-extrinsic stenosis can be seen. For instance, the degree of extrinsic stenosis can be so severe and short-segmented as to remove the typically seen feature of extrinsic stenosis tapering, giving a false appearance of a rounded filling defect on 3D reconstructions (Fig 7).

However, source images would reveal an abrupt change in caliber related to brain parenchyma and not primary intrinsic stenosis lead points. One useful means of distinguishing severe extrinsic-versusintrinsic stenosis is the continuity of the stenosis in question. 
Specifically, extrinsic stenosis is typically continuous in length owing to its uniform brain parenchymal extension into the sinus. In contrast, intrinsic stenosis may manifest as discontinuous points of stenosis, depending on the distribution and number of contributory arachnoid granulations.

Some patients may have features of both intrinsic and extrinsic stenosis. This was seen in 17 of the patients in the idiopathic intracranial hypertension cohort, noting that such a mixed appearance was not identified in any of the patients in the pulsatile tinnitus cohort. All these cases had the appearance of clustered arachnoid granulations located either proximal or distal to the area of otherwise-prominent extrinsic stenosis. Arachnoid granulations can demonstrate compensatory enlargement to promote further reabsorption of CSF in the setting of an underlying dural venous sinus stenosis. ${ }^{12}$ Thus, in patients with arachnoid granulations about the margins of a prominent sinus narrowing from overlying parenchyma, the authors considered these sinuses as having overall extrinsic stenosis (Fig 8).

The presence of internal jugular vein diverticula or prominent emissary veins was uniformly distributed across both cohorts, presumably related to pressure-induced vessel dilation and collateral redistribution in the setting of stenosis. The prominence of emissary veins may not be uniquely specific to the diagnosis of idiopathic intracranial hypertension, given their similar prominence in patients with both idiopathic intracranial hypertension and isolated venous pulsatile tinnitus, as previously reported with regard to the occipital emissary vein. ${ }^{13}$ In the pulsatile tinnitus cohort, management of the underlying transstenotic gradient with stent placement alone was sufficient in resolving patients' tinnitus symptomology without the need for additional internal jugular bulb diverticulum or emissary vein embolization. Given the similar distribution of emissary vein prominence and internal jugular bulb diverticula across both cohorts, the resolution of tinnitus following transstenotic gradient resolution adds support to stent placement while avoiding unnecessary embolization of prominent emissary veins, especially in the absence of an associated saccular venous aneurysm. Nevertheless, in the authors' experience, a subset of patients not included in this study in whom the absence of a notable venous sinus stenosis or pressure gradient was recorded demonstrated benefit from embolization of such corollary findings if the side of pulsatile tinnitus corroborated with the side of the diverticulum or emissary vein.

Cerebellar tonsils were either at or slightly below the foramen magnum level in a similar distribution across both cohorts. A notably increased presence of marked optic nerve tortuosity, empty sella, and cephalocele identification in the idiopathic intracranial hypertension cohort relative to the pulsatile tinnitus cohort suggests that these imaging manifestations are more specific to idiopathic intracranial hypertension rather than their incidental presence in the setting of lateral sinus stenosis, as previously reported with regard to the empty sella. ${ }^{14}$ Similarly, a notably increased prevalence of saccular venous aneurysm in the pulsatile tinnitus cohort relative to idiopathic intracranial hypertension suggests that aneurysm formation may be more specific to pulsatile tinnitus. Despite such trends, the overlapping of all corollary findings assessed in this study between both cohorts adds further credence to the notion that a clinical spectrum between pulsatile tinnitus and idiopathic intracranial hypertension exists. On the basis of the results of this study, the authors propose that the evaluation of stenosis subtype be considered a similarly important diagnostic finding alongside the other corollary imaging findings discussed in this study.

\section{CONCLUSIONS}

In this cohort, most patients with idiopathic intracranial hypertension had extrinsic venous sinus stenosis, and most patients with pulsatile tinnitus had intrinsic venous sinus stenosis. The authors propose that the reporting of venous sinus stenosis subtype as either intrinsic or extrinsic be considered in standardized MRV reporting. Awareness and reporting of these subtypes on diagnostic imaging may reduce underrecognition of potentially symptomatic venous sinus stenoses. This, in turn, may allow improved multidisciplinary discussions and appropriately tailored management in patients with idiopathic intracranial hypertension and pulsatile tinnitus, accordingly.

Disclosures: Vaishnavi Kishore-UNRELATED: Employment: GE Healthcare. Raphael Doustaly-UNRELATED: Employment: GE Healthcare. Athos PatsalidesUNRELATED: Consultancy: MicroVention, Penumbra, money paid to Weill Cornell Medical College.

\section{REFERENCES}

1. Dinkin MJ, Patsalides A. Venous sinus stenting for idiopathic intracranial hypertension: where are we now? Neurol Clin 2017;35:59-81 CrossRef Medline

2. Patsalides A, Oliveira C, Wilcox J, et al. Venous sinus stenting lowers the intracranial pressure in patients with idiopathic intracranial hypertension. J Neurointerv Surg 2019;11:175-78 CrossRef Medline

3. Toscano S, Lo Fermo S, Reggio E, et al. An update on idiopathic intracranial hypertension in adults: a look at pathophysiology, diagnostic approach and management. J Neurol 2020 May 27. [Epub ahead of print] CrossRef Medline

4. Johnston I. The historical development of the pseudotumor concept. Neurosurg Focus 2001;11:E2 CrossRef Medline

5. Hewes D, Morales R, Raghavan P, et al. Pattern and severity of transverse sinus stenosis in patients with pulsatile tinnitus associated with sigmoid sinus wall anomalies. Laryngoscope 2020;130:1028-33 CrossRef Medline

6. Pekcevik R, Pekcevik Y. Anatomy of the posterior fossa emissary veins and their clinical importance. In: Proceedings of the Annual Meeting of the European Congress of Radiology, Vienna, Austria. June 7-11, 2013

7. Bergman RA, Tubbs RS, Shoja MM, et al. Dural venous sinuses. In: Bergman RA, Tubbs RS, Shoja MM, et al. Bergman's Comprehensive Encyclopedia of Human Anatomic Variation. John Wiley \& Sons; 2019:775-99

8. Sivasankar R, Pant R, Indrajit IK, et al. Imaging and interventions in idiopathic intracranial hypertension: a pictorial essay. Indian $J$ Radiol Imaging 2015;25:439-44 CrossRef Medline

9. Newman CW, Jacobson GP, Spitzer JB. Development of the Tinnitus Handicap Inventory. Arch Otolaryngol Head Neck Surg 1996;122:143-48 CrossRef Medline

10. Boddu SR, Gobin P, Oliveira C, et al. Anatomic measurements of cerebral venous sinuses in idiopathic intracranial hypertension patients. PLoS One 2018;13:e0196275 CrossRef Medline 
11. Lenck S, Vallee F, Labeyrie MA, et al. Stenting of the lateral sinus in idiopathic intracranial hypertension according to the type of stenosis. Neurosurgery 2017;80:393-400 CrossRef Medline

12. Mondejar V, Patsalides A. The role of arachnoid granulations and the glymphatic system in the pathophysiology of idiopathic intracranial hypertension. Curr Neurol Neurosci Rep 2020;20:20 CrossRef Medline
13. Hedjoudje A, Piveteau A, Gonzalez-Campo C, et al. The occipital emissary vein: a possible marker for pseudotumor cerebri. AJNR Am J Neuroradiol 2019;40:973-78 CrossRef Medline

14. Zetchi A, Labeyrie M-A, Nicolini E, et al. Empty sella is a sign of symptomatic lateral sinus stenosis and not intracranial hypertension. AJNR Am J Neuroradiol 2019;40:1695-1700 CrossRef Medline 